Discrete Comput Geom 30:453-458 (2003)

DOI: $10.1007 / \mathrm{s} 00454-003-2818-8$

\title{
The Number of Cylindrical Shells
}

\author{
Olivier Devillers \\ INRIA, Unité de Recherche Sophia Antipolis, \\ BP93, 06902 Sophia-Antipolis, France \\ Olivier.Devillers@sophia.inria.fr
}

\begin{abstract}
Given a set $P$ of $n$ points in three dimensions, a cylindrical shell (or zone cylinder) is formed by two circular cylinders with the same axis such that all points of $P$ are between the two cylinders. We prove that the number of cylindrical shells enclosing $P$ passing through combinatorially different subsets of $P$ has size $\Omega\left(n^{3}\right)$ and $O\left(n^{4}\right)$ (the previously known bound was $\left.O\left(n^{5}\right)\right)$. As a consequence, the minimum enclosing shell can be found in $O\left(n^{4}\right)$ time.
\end{abstract}

\section{Introduction}

Consider a set $P$ of $n$ points in three dimensions. A cylindrical shell is formed by the space between two coaxial circular cylinders in three dimensions. The shell is said to enclose $P$ if the set $P$ is between the two cylinders. The difference between the radii of the two cylinders is the width of the shell.

Finding the minimum-width shell enclosing $P$ is an important metrology problem. To control the quality of cylindrical objects (e.g., mechanical pieces, pistons, etc.), points are probed on the boundary of the cylinder and the width of the minimum-width enclosing shell is a recognized measure of the quality of the piece [1]. Computing the minimumwidth enclosing shell has already received attention in the literature. In the special case of small width, Devillers and Preparata compute a provably good approximation of the minimum-width enclosing shell by reducing the problem to linear programming [5]. Agarwal et al. compute a solution which approximates the optimum up to a constant factor in quadratic time [2]. Har-Peled and Varadarajan propose to compute an $(1+\varepsilon)$ approximation in time linear in $n$ and exponential in $1 / \varepsilon[6]$.

Few exact algorithms have been developed for computing the minimum-width enclosing shell. In general position, six points define a constant number of shells having these six points on their boundary (less than 150) [4], thus a naive algorithm will have 
$O\left(n^{7}\right)$ complexity. Agarwal et al. [2] have proposed a reduction to convex hull in ten dimensions and deduced an algorithm of complexity $O\left(n^{5}\right)$.

In this paper we prove new upper and lower bounds for the number of combinatorially different enclosing cylindrical shells for a set of $n$ points in three-dimensional space. Using the new upper bound, the complexity for the exact algorithm of Agarwal et al. computing the smallest-width enclosing shell can be reduced to $O\left(n^{4}\right)$.

\section{Lower Bound Example}

Theorem 1. Given $n$ points in three dimensions, the number of combinatorially different enclosing shells is $\Omega\left(n^{3}\right)$.

Proof. We construct below a set of $3 n$ points with more than $n^{3}$ combinatorially different enclosing shells. We consider three sets of $n$ points:

$$
\begin{array}{rlrl}
p_{i} & =\left(\frac{i}{n}, 0,0\right), & 1 \leq i \leq n, \\
q_{i}=\left(\frac{i}{n}, 0, \zeta\right), & 1 \leq i \leq n, \\
r_{i}=\left(x_{i}, \frac{i}{n}, 0\right), & 1 \leq i \leq n,
\end{array}
$$

such that the $r_{i}$ belong to the circle $\mathcal{C}$ in plane $z=0$ with center $c=\left(3, \frac{1}{2}, 0\right)$ and radius 6 , and $\zeta$ is some positive parameter to be chosen later (see Fig. 1).

Then for any triple $(i, j, k)$ we construct a shell such that the internal cylinder is tangent to line $y=z=0$ at $p_{i}$ and to line $y=\zeta-z=0$ at $q_{j}$ and such that the external cylinder contains and is tangent to circle $\mathcal{C}$ at $r_{k}$.
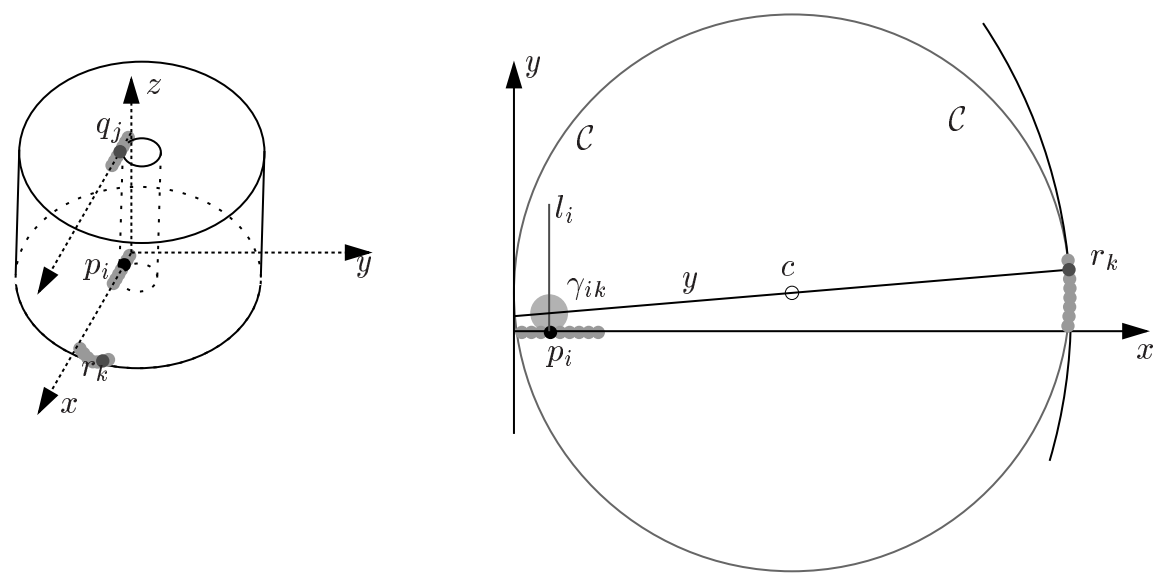

Fig. 1. $\Omega\left(n^{3}\right)$ lower bound example. 
To guarantee the tangencies at points $p_{i}$ and $q_{j}$, it is enough to have a cylinder with the axis parallel to $p_{i} q_{j}$ that passes through a point of the line $l_{i}$ through $p_{i}$ and parallel to the $y$ axis. We choose that point to be the intersection point $\gamma_{i k}$ of the line through $c$ and $r_{k}$ and the line $l_{i}$. Then the cylinder with axis $\alpha_{i j k}$, passing through $p_{i}$, also passes through $q_{j}$ and is tangent to the plane $O x z$, so that points $p_{l}, l \neq i$, and $q_{l}, l \neq j$, are outside the cylinder.

We consider now the cylinder with the same axis $\alpha_{i j k}$ passing through $r_{k}$. First we remark that, in the horizontal plane, the circle of center $\gamma_{i k}$ passing through $r_{k}$ is tangent externally to $\mathcal{C}$ since its radius is bigger than the radius of $\mathcal{C}$. Since the cylinder is not vertical, its intersection with the horizontal plane is not a circle but an ellipse, although, by choosing $\zeta$ large enough, it is possible to make this ellipse close enough to a circle and to preserve the property that $r_{k}$ is on the cylinder and the $r_{l}, l \neq k$, are inside the cylinder.

\section{Projection Theorem}

We prove in this section that the convex hull of certain special configurations of points in $d$ dimensions cannot reach the worst-case complexity of $O\left(n^{\lfloor d / 2\rfloor}\right)$. These special configurations are point sets in a $d$-dimensional space of size $2 n$. They are constructed by taking $n$ points in a hyperplane and adding to them their parallel projection on another hyperplane. In such configurations, the convex hull in $d$ dimensions is the extrusion of the convex hull in one of the hyperplanes and has complexity $O\left(n^{\lfloor(d-1) / 2\rfloor}\right)$. More precisely, we have:

Theorem 2. Let $\pi, \pi^{\prime}$ be two hyperplanes in $\mathbb{R}^{d}$ and let $\vec{v}$ be a vector of $\mathbb{R}^{d}$ which is not parallel to either $\pi$ or $\pi^{\prime}$. Let $\mathcal{U} \subset \pi$ be a finite set of $n$ points all lying on the same side of $\pi^{\prime}$ and let $\mathcal{U}^{\prime} \subset \pi^{\prime}$ be the projection of $\mathcal{U}$ on $\pi^{\prime}$ along direction $\vec{v}$. Let $\mathcal{V}=\mathcal{U} \cup \mathcal{U}$. The convex hull $\mathrm{CH}(\mathcal{V})$ of $\mathcal{V}$ has the same asymptotic complexity as $\mathrm{CH}(\mathcal{U})$, that is, $O\left(n^{\lfloor(d-1) / 2\rfloor}\right)$.

Proof. The space is divided into four quadrants by the hyperplanes $\pi$ and $\pi^{\prime}$ (see Fig. 2). Three of these quadrants are obviously separated from $\mathcal{V}$ by $\pi$ or $\pi^{\prime}$ and thus cannot intersect $C H(\mathcal{V})$. Consider a face $F$ of $C H(\mathcal{V})$ in the fourth quadrant; we first claim that $\vec{v}$ is parallel to $F$. We denote by $H$ a hyperplane supporting $F$ and by $n_{H}$ the normal to $H$. Let $x$ be a vertex of $F$ in $\pi$ and let $x^{\prime}$ be its projection on $\pi^{\prime}$. Similarly, let $y^{\prime}$ a vertex of $F$ in $\pi^{\prime}$ and let $y$ be its projection on $\pi$. Since $H$ is a supporting hyperplane we must have $x^{\prime}$ and $y$ on the same side of $H$, that is, $\operatorname{sign}\left(\overrightarrow{x x^{\prime}} \cdot n_{H}\right)=\operatorname{sign}\left(\overrightarrow{y^{\prime} y} \cdot n_{H}\right)$, but since $\overrightarrow{x x^{\prime}}$ and $\overrightarrow{y^{\prime} y}$ are collinear to $\vec{v}$ and in opposite directions, we get that $\vec{v}$ is parallel to $H$.

In fact we have that the faces of $C H(\mathcal{V})$ are either faces of $\mathrm{CH}(\mathcal{U})$, faces of $\mathrm{CH}\left(\mathcal{U}^{\prime}\right)$ or faces linking a face of $\mathrm{CH}(\mathcal{U})$ to its projection in $\mathrm{CH}\left(\mathcal{U}^{\prime}\right)$. Thus the total number of faces of $\mathrm{CH}(\mathcal{V})$ is three times the number of faces of $\mathrm{CH}(\mathcal{U})$.

Another proof of this result can be obtained using a projective transformation of the space $\theta$ sending the plane generated by $\pi \cap \pi^{\prime}$ and $\vec{v}$ to infinity. Such a transformation 


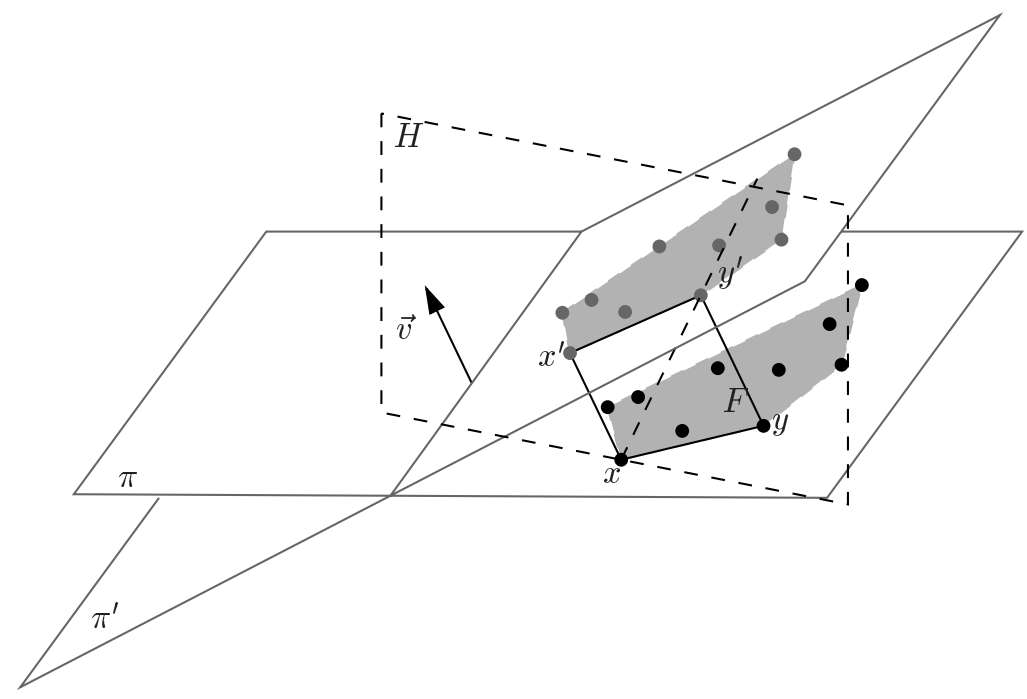

Fig. 2. For the proof of Theorem 2 .

does not change the combinatorial structure of a convex hull while at the same time making the two hyperplanes parallel and leaving $\mathcal{U}^{\prime}$ a projection of $\mathcal{U}$.

Unfortunately, Theorem 2 does not generalize (as stated) to the case where $\pi$ and $\pi^{\prime}$ are $k$-flats with $k \leq d-2$. For example, if $\mathcal{U}=\left\{p_{i}=\left(i, i^{2}, 0,0\right) ; 1 \leq i \leq n\right\}$ and $\mathcal{U}^{\prime}=\left\{p_{j}^{\prime}=\left(0,0, j, j^{2}\right) ; 1 \leq j \leq n\right\}$, then the $O\left(n^{2}\right)$ simplices $\left(p_{i}, p_{i+1}, p_{j}^{\prime}, p_{j+1}^{\prime}\right)$ are convex hull faces.

\section{Minimum-Width Cylindrical Shell Algorithm}

Given a set $\mathcal{S}$ of $n$ three-dimensional points Agarwal et al. [2, page 310] exhibit a transformation mapping a three-dimensional point $p=(x, y, z)$ in two half-spaces $P^{\star}$ and $P^{\dagger}$ in ten dimensions:

$$
\begin{aligned}
P^{\star}: \varphi_{9} \leq & \left(x^{2}+y^{2}\right)+2 x \varphi_{1}+2 y \varphi_{2}+2 z \varphi_{3}-2 x y \varphi_{4}-2 x z \varphi_{5}-2 y z \varphi_{6} \\
& +\left(y^{2}+z^{2}\right) \varphi_{7}+\left(x^{2}+z^{2}\right) \varphi_{8}, \\
P^{\dagger}: \varphi_{10} \geq & \left(x^{2}+y^{2}\right)+2 x \varphi_{1}+2 y \varphi_{2}+2 z \varphi_{3}-2 x y \varphi_{4}-2 x z \varphi_{5}-2 y z \varphi_{6} \\
& +\left(y^{2}+z^{2}\right) \varphi_{7}+\left(x^{2}+z^{2}\right) \varphi_{8},
\end{aligned}
$$

where $\varphi_{1}, \ldots \varphi_{10}$ are the coordinates in ten dimensions. They prove that the complexity of the intersection of the $2 n$ half-spaces $P^{\star}$ and $P^{\dagger}$ is a bound on the number of combinatorially distinct enclosing shells. Moreover, computing this intersection can be done in time proportional to its worst-case size [3], thus enabling us to enumerate all possible shells and thereby find the minimum-width one. Since the complexity of the intersection of $2 n$ half-spaces in ten dimensions is $O\left(n^{5}\right)$, the same complexity is obtained for the minimum-width enclosing shell algorithm. 
The problem of intersecting half-spaces can be transformed to a convex hull problem through the well-known duality between points and hyperplanes, provided that a point inside the intersection of half-spaces is known. Here we note that for some $\alpha$ large enough, the point $(0,0,0,0,0,0,0,0,0, \alpha)$ is certainly strictly inside the half-spaces $P^{\star}$ and $P^{\dagger} .{ }^{1}$ By a translation by that vector, the hyperplane

$$
0=\psi_{0}+\sum_{i=1}^{10} \psi_{i} \varphi_{i}
$$

is transformed to the hyperplane

$$
0=\left(\psi_{0}+\psi_{10} \alpha\right)+\sum_{i=1}^{10} \psi_{i} \varphi_{i},
$$

where $\varphi_{i}$ are the coordinates in the new frame. This equation can be normalized by dividing it by $\psi_{8}$ and by duality we get the point

$$
\left(\frac{\psi_{0}+\psi_{10} \alpha}{\psi_{8}}, \frac{\psi_{1}}{\psi_{8}}, \frac{\psi_{2}}{\psi_{8}}, \frac{\psi_{3}}{\psi_{8}}, \frac{\psi_{4}}{\psi_{8}}, \frac{\psi_{5}}{\psi_{8}}, \frac{\psi_{6}}{\psi_{8}}, \frac{\psi_{7}}{\psi_{8}}, \frac{\psi_{9}}{\psi_{8}}, \frac{\psi_{10}}{\psi_{8}}\right)
$$

Through this process, a three-dimensional point $p=(x, y, z)$ gives two half-spaces $P^{\star}$ and $P^{\dagger}$ in ten dimensions and then by duality two points $p^{\star}$ and $p^{\dagger}$ in ten dimensions:

$$
\begin{aligned}
p^{\star}= & \left(\frac{x^{2}+y^{2}}{x^{2}+z^{2}}, \frac{2 x}{x^{2}+z^{2}}, \frac{2 y}{x^{2}+z^{2}}, \frac{2 z}{x^{2}+z^{2}},\right. \\
& \left.\frac{-2 x y}{x^{2}+z^{2}}, \frac{-2 x z}{x^{2}+z^{2}}, \frac{-2 y z}{x^{2}+z^{2}}, \frac{y^{2}+z^{2}}{x^{2}+z^{2}}, \frac{1}{x^{2}+z^{2}}, 0\right) \\
p^{\dagger}= & \left(\frac{x^{2}+y^{2}}{x^{2}+z^{2}}+\frac{\alpha}{x^{2}+z^{2}}, \frac{2 x}{x^{2}+z^{2}}, \frac{2 y}{x^{2}+z^{2}}, \frac{2 z}{x^{2}+z^{2}},\right. \\
& \left.\frac{-2 x y}{x^{2}+z^{2}}, \frac{-2 x z}{x^{2}+z^{2}}, \frac{-2 y z}{x^{2}+z^{2}}, \frac{y^{2}+z^{2}}{x^{2}+z^{2}}, 0, \frac{1}{x^{2}+z^{2}}\right) \\
= & p^{\star}+\frac{1}{x^{2}+z^{2}}(\alpha, 0,0,0,0,0,0,0,-1,1) .
\end{aligned}
$$

We remark that $p^{\star}$ belongs to the half-hyperplane $\pi^{+}: \varphi_{10}=0, \varphi_{9} \geq 0$ and that $p^{\dagger}$ is the projection of $p^{\star}$ on $\pi^{\prime}: \varphi_{9}=0$ along direction $\vec{v}=(\alpha, 0,0,0,0,0,0,0,-1,1)$. The hypotheses for Theorem 2 are satisfied and we conclude that the complexity of the convex hull of the set of $2 n$ points formed by the $p^{\star}$ and $p^{\dagger}(p \in \mathcal{S})$ can be reduced to $O\left(n^{4}\right)$. The convex hull can be actually computed in that time by computing the hull of points $p^{\star}[3]$ and reconstructing the facets linking $\pi$ and $\pi^{\prime}$ from that hull. We can thus state the following theorem, improving the previous result by a factor of $n$ :

\footnotetext{
${ }^{1}$ This is the case if the origin is chosen outside $\mathcal{S}$ and if $\alpha$ is larger than the square of the distance between the origin and its farthest neighbor in $\mathcal{S}$.
} 
Theorem 3. Given a set $\mathcal{S}$ of $n$ points in $\mathbb{R}^{3}$, a minimum-width cylindrical shell containing $\mathcal{S}$ can be computed in $O\left(n^{4}\right)$ time.

\section{Open Problems}

Our lower bound of $\Omega\left(n^{3}\right)$ applies to combinatorially different cylindrical shells enclosing a set of $n$ points without consideration of width. We may ask if the bound applies also to the number of local or global minima of the width function on the set of enclosing shells.

Another obvious problem is to reduce the gap between $\Omega\left(n^{3}\right)$ and $O\left(n^{4}\right)$.

\section{Acknowledgments}

Thanks to Menelaos Karavelas and Hazel Everett for their help in preparing this note.

\section{References}

1. ISO/DIS 12180. Geometrical product specifications (gps) - cylindricity, 2000.

2. Pankaj K. Agarwal, Boris Aronov, and Micha Sharir. Exact and approximation algorithms for minimumwidth cylindrical shells. Discrete Comput. Geom., 26:307-320, 2001.

3. Bernard Chazelle. An optimal convex hull algorithm in any fixed dimension. Discrete Comput. Geom., 10:377-409, 1993.

4. Olivier Devillers, Bernard Mourrain, Franco P. Preparata, and Philippe Trebuchet. On circular cylinders by four or five points in space. Discrete Comput. Geom., 29:83-104, 2003.

5. Olivier Devillers and Franco P. Preparata. Evaluating the cylindricity of a nominally cylindrical point set. In Proc. 11th ACM-SIAM Sympos. Discrete Algorithms, pages 518-527, 2000.

6. Sariel Har-Peled and Kasturi R. Varadarajan. Approximate shape fitting via linearization. In Proc. 42nd Anпu. IEEE Sympos. Found. Comput. Sci., 2001.

Received July 30, 2001, and in revised form August 31, 2002, and September 25, 2002.

Online publication July 25, 2003. 